\title{
Cervical screening adherence in the era of HPV vaccination: how low is too low?
}

Chris T. Bauch, $\mathrm{PhD}^{1 *}$, Meng Li, $\mathrm{MSc}^{2}$, Gretchen Chapman, $\mathrm{PhD}^{2}$, Alison P. Galvani, $\mathrm{PhD}^{3}$

${ }^{1}$ Department of Mathematics and Statistics, University of Guelph, Guelph, ON, Canada

2 Department of Psychology, The State University of New Jersey, Piscataway, NJ, USA

${ }^{3}$ Department of Epidemiology and Public Health, Yale University School of Medicine, New Haven, Connecticut, USA

For submission to The Lancet Infectious Diseases as a Personal View contribution

Original version submitted 4 March 2009

Revised version submitted 21 September 2009

Main text word count: 2,996 excluding references

* to whom correspondence should be sent.

Telephone: +001 (519) 824-4120 ext. 53079

Fax: $\quad+001(519)$ 837-0221

Email: $\quad$ cbauch@uoguelph.ca

Address: Department of Mathematics and Statistics

University of Guelph

Guelph, ON N1G 2W1

Canada 
The recently licensed human papillomavirus (HPV) vaccine prevents infection by two major oncogenic HPV types. However, continued screening in vaccinated women is necessary to prevent cancers caused by high-risk types not included in the vaccine. In the vaccine era, there is new concern that vaccinated women will reduce their rate of screening due to an exaggerated sense of protection from the vaccine. Some have suggested this could paradoxically lead to an increase in the incidence of cervical cancer. Here, we present a simple mathematical model suggesting that this outcome is unlikely. This result applies under a wide range of possible vaccine coverage levels and screening programme effectiveness. In populations that currently have highly effective cervical screening programmes, screening rates in vaccinated women have less room to fall before cervical cancer incidence starts to increase in the vaccine era, although the required declines are still very steep. 


\section{Introduction}

Recently introduced human papillomavirus (HPV) vaccines are highly effective in preventing infection by the predominant HPV types 16 and 18 that cause $70 \%$ of cervical cancer cases (1). The remaining 30\% of cases are caused by high-risk (oncogenic) HPV types not included in the vaccine and which can thereby only be prevented through continued screening (2). Cervical screening is invasive and costly and it has been argued that screening frequencies are too high in some groups (3). However, a new concern that has emerged in the vaccine era is that vaccinated women will reduce their frequency of screening too much, due to an exaggerated sense of security from being vaccinated (4).

The landscape of cervical cancer prevention and treatment is changing dramatically, not only because of the HPV vaccine but also because of new screening technologies. Highly sensitive molecular tests for the DNA of high-risk HPV types are quickly becoming mainstream. Maintaining annual Pap tests to detect the $30 \%$ of cervical cancers originating from non-16/18 HPV types will likely prove inefficient in the vaccine era. However, integrating HPV DNA testing into the cervical screening platform can improve programme effectiveness by increasing sensitivity for detection of cervical intraepithelial neoplasias, enabling screening for vaccine failures, and permitting longer screening intervals $(5,6)$.

"Policy resistance" has been defined as the "tendency for interventions to be defeated by the system's response to the intervention itself" (7). An outcome where screening rates in vaccinated women drop so much that the number of cervical cancer cases thereby created exceeds the number prevented through vaccination is an extreme example of policy resistance. We define the "screening threshold" as the critical screening rate at which this occurs. Being able to estimate the screening threshold would be valuable from both public health and clinical perspectives.

Mathematical models can be used to evaluate the potential risks and benefits of different cervical screening and HPV vaccination strategies. Numerous models have simulated the transmission dynamics and pathogenesis of HPV as well as the impacts of 
HPV vaccination (8-18). These models have varied widely in how disease pathogenesis and HPV type structure are represented.

A key challenge in applying mathematical models to HPV infection and cervical screening has been the great uncertainty in the natural history of disease among different HPV types. Most available data concerns type 16, which progresses more quickly and is more pathogenic than other types $(2,19)$. The parameters describing the infection risk and progression of other HPV types have usually been inferred indirectly, often through large curve-fitting experiments that are contingent upon the structure of the model being used. Thus, a variety of different models concordant with currently available epidemiological data can produce different projections because of the uncertainty in parameter values describing HPV pathogenesis $(8,11-13,17,20)$.

In the context of such large uncertainties, simple mathematical models can provide intuitive context to the problem, or define clear thresholds that have to be surpassed to meet public health goals (21). In the context of cervical screening in particular, a simple model can be used to ask the fundamental question: how much can cervical screening adherence in vaccinated women drop before the incidence of cervical cancer will start to increase? A simple model may provide insight to this question, andunlike more complex models - can better clarify fundamental relationships among the key variables that affect the screening threshold.

A potential limitation of simple models is that their simplifying assumptions can restrict their usefulness when a "best guess" projection is needed. However, this limitation can often be circumvented when a conservative (upper or lower bound) estimate is all that is needed. For instance, if simpler models are known to be valid at upper or lower credible values of certain crucial parameters it may be possible to thereby obtain conservative (e.g. upper or lower credible) estimates of crucial thresholds. Here, we adopt such an approach by analyzing a simple mathematical model that yields a conservative estimate of the cervical screening threshold in vaccinated women below which policy resistant outcomes emerge.

\section{Mathematical model}


We model a population of women who are infected with at least one high-risk HPV type and are thus defined to be at risk of developing cervical cancer. We suppose that $H$ women per year enter the at-risk population due to infection by high-risk types. The per capita rate at which they are removed from the at-risk population through screening (and follow-up where needed) is $s_{\text {pre }}$ per year in the pre-vaccine era; $s_{\text {pre }}$ is the rate of efficacious screening, hence it is high in populations where both the rate of screening and the quality of cytology and follow-up of abnormal results are adequate. Processes other than screening - primarily natural clearance of infection but also mortality and benign hysterectomies - remove women from the at-risk population at per capita rate $r$ per year.

We suppose that a fraction $f$ of girls are vaccinated, and that vaccination reduces the rate at which they become infected by a factor $e$, where $e$ is the realized effectiveness of vaccination programmes for preventing infection. Therefore unvaccinated women enter the at-risk population at rate $H(1-f)$ and vaccinated women enter the at-risk population at rate $H f(1-e)$. We suppose that new guidelines and technologies in the vaccine era change the rate of removal through screening from $s_{\text {pre }}$ to $s_{\text {post. }}$. Also, we suppose vaccinated women change their frequency of cervical screening visits by a factor $x$, such that the rate of removal from the at-risk population through screening and followup becomes $x \cdot S_{\text {post. }}$. For instance, when $x=0.5$ vaccinated women seek screening half as often. Therefore, a fraction (1-f) of unvaccinated women are removed through screening at rate $s_{\text {post }}$ in the vaccine era, while a fraction $f$ are removed at rate $s_{\text {post }} x$. The model equations appear in the Appendix.

If screening adherence for vaccinated women declines too much in the vaccine era, then the size of the at-risk population could increase in the vaccine era. In the Appendix we solve the model to show that the threshold value of $x$ below which this occurs is

$$
x^{*}=1-\frac{1}{f}\left(1-\frac{s_{\text {pre }}}{s_{\text {post }}}\right)-e\left(\frac{r+s_{\text {pre }}}{s_{\text {post }}}\right) .
$$

The values of $e, r, s_{\text {pre }}$ and $f$ can be estimated from the literature. A recent study in the United States finds a prevalence of $\sim 1.5 \%$ for type $16, \sim 0.8 \%$ for type 18 , and $\sim 15.2 \%$ for any high-risk type (22). Clinical trial data indicate that the vaccine is at least $95 \%$ efficacious in preventing infection by types 16 and 18 (23). Our baseline assumption 
is lifelong vaccine-derived immunity and no type replacement. Hence, a baseline value for vaccine effectiveness $e$ can be approximated as the type-specific vaccine efficacy weighted by type prevalence, yielding $e=0.95 \times(1.5+0.8) / 15.2=0.14$. We note that most other surveys have found higher type 16/18 prevalence (24), hence using the lower type 16/18 prevalence reported in Ref. 22 can be considered conservative. The rate $r$ is dominated by natural clearance, which has been previously estimated as $r=0.83$ per year for types 16 and 18, hence we adopt this value as our baseline estimate. The screening rate has been estimated to be $44 \%$ per year under opportunistic screening programmes in the United States where quality of cytology and follow-up are high. Hence, we take $s_{\text {pre }}=0.44 /$ year under the baseline pre-vaccine "opportunistic screening" scenario $(25,26)$

The value of $s_{\text {post }}$ cannot be known, but it is reasonable to assume that $s_{\text {post }}>S_{\text {pre }}$, $(5,6)$. Under the conservative assumption that $s_{\text {post }}=s_{\text {pre }}=s$, Equation (1) reduces to (2) $\quad x^{*}=1-e \frac{(r+s)}{s}$.

The way that $x^{*}$ depends on $e, r$, and $s$ in Equation (2) is visualized in Figure 1. In the neighbourhood of the baseline parameter values, variation in $e$ or $r$ has a larger impact on $x^{*}$ than variation in $s$. This implies that the screening threshold is significantly affected by type $16 / 18$ prevalence. At the baseline parameter values, we calculate $x^{*}=0.61$ from Equation (2). This means the rate at which vaccinated women seek screening would have to drop by at least $39 \%$ before the size of the at-risk population (women infected with high-risk HPV but not yet screened) would increase in the vaccine era.

The estimate $x^{*}=0.61$ is contingent upon very specific parameter values, hence it is neither robust nor applicable to all populations. To apply the model to a broader range of populations, we added an "organized screening" scenario, in which $s_{\text {pre }}$ is higher than under the baseline "opportunistic screening" scenario (14), and a "limited screening" scenario, in which $s_{\text {pre }}$ is lower than the baseline, as might occur in countries where access to cytology screening is limited, and/or where the quality of cytology and management of abnormal cases are inadequate. The "limited screening" scenario is relevant to low-income countries, for which cervical cancer vaccination programmes may be on the horizon (27). 
We also carried out a probabilistic uncertainty analysis on Equation (1) to test model robustness and understand the impact of data uncertainty. This involved defining plausible lower and upper bounds for $e, r, s_{\text {pre }}$, and $s_{\text {post }}$ and sampling repeatedly from the resulting intervals. We assumed $e \in[0.09,0.25]$ and $r \in[0.5 / \mathrm{yr}, 1.5 / \mathrm{yr}]$ for all three screening scenarios (see Webappendix for details). The intervals for $s_{\text {pre }}$ differed for the three screening scenarios and are given in Table 1. As noted above, it is reasonable to suppose $s_{\text {poss }}>s_{\text {pre }}$. We obtained a value of $s_{\text {post }}$ from each sample of $s_{\text {pre }}$ by multiplying the sampled value of $s_{\text {pre }}$ by a factor $h>1$, which was a random number selected from $h \in$ $[1.0,1.2]$. We then sampled repeatedly from these four intervals to generate 5,000 realized parameter sets; calculated $x^{*}$ from Equation (1) for each parameter set; and determined the median and standard deviation of all realized values of $x^{*}$. This was done for both $f=0.3$ and $f=0.7$.

The results of the probabilistic uncertainty analysis under the three screening scenarios appear in Table 1. In the "limited screening" scenario the median threshold is zero for both values of $f$, meaning that vaccinated women could stop screening altogether and there would not be an increase in cervical cancer incidence in the population. The median thresholds are also relatively low in the "opportunistic" and "organized" screening scenarios for both values of $f$, requiring steep declines of $49 \%$ or more in screening adherence before policy resistance emerges. The large variability in $x^{*}$ across the realizations, which is largely driven by uncertainty in parameters $e$ and $r$, should be interpreted in light of the fact that Equation (1) is a conservative (upper bound) estimate of the true threshold, as we will attempt to establish in the Discussion section.

The median threshold increases as the pre-vaccine screening removal rate $s_{\text {pre }}$ increases across these three screening scenarios (this is also apparent in Figure 1b). Hence, in populations with excellent screening programmes in the pre-vaccine era, policy resistance emerges for smaller declines in screening adherence in the vaccine era. This seemingly counterintuitive result reflects that fact that women in the pre-vaccine era undergoing a high screening rate with high-quality cytology and adequate management of abnormal cases will receive a smaller incremental reduction in cancer risk from also having the vaccine, and therefore screening rates in the vaccine era would have to remain high to prevent the at-risk population from growing. On the other hand, populations with 
less effective screening programmes in the pre-vaccine era-such as many low-income countries - would experience large benefits from the introduction of vaccination programmes, and therefore screening rates could decline more before policy resistance emerges.

\section{Discussion and model extensions}

This model makes a number of simplifying assumptions. For instance, it ignores type structure, type replacement, varying pathogenicity and progression rates among types, herd immunity, and heterogeneity in screening rates within populations. Here, we argue that most of these assumptions have the convenient dual purpose of both simplifying the model and ensuring that the resulting estimate of the screening threshold is conservative. We also relax some of these assumptions in extended versions of the model to explore how the screening threshold thereby changes.

Equation (1) provides a screening threshold when the outcome of interest is infection by any high-risk type. Several lines of evidence suggest that the screening threshold will be significantly lower when the outcome of interest is cervical cancer incidence. Firstly, Equation (1) does not distinguish between pathogenicity of types. However types 16 and 18 are more pathogenic, accounting for approximately $70 \%$ of all cervical cancer cases despite constituting only $15 \%$ of high-risk infections $(2,28)$. Therefore, because the vaccine is highly efficacious against types 16 and 18, the screening threshold when cervical cancer incidence is the outcome of interest should be well below the screening threshold when high-risk infection is the outcome of interest.

Compensatory effects may partially close this gap. For example, the incidence of cervical cancer due to non-16/18 types may increase as an artifact of medical management: before vaccination, the removal of pre-cancerous lesions associated with types 16/18 would also have removed any lesions associated with other slowerprogressing types. However, in the vaccine era, these removals will happen less often and so lesions from other high-risk types could continue progressing (9). There is also a theoretical possibility of type replacement, whereby non-16/18 types fill the empty ecological niche create by removal of types 16/18 (18). (However there is lack of 
empirical evidence for this effect, and this outcome is unlikely due to the genetic diversity of HPV types.)

For these compensatory factors to close the gap completely, the prevalence of other high-risk types and/or their pathogenicity would have to increase enormously in the vaccine era to make up for large difference in pathogenicity between types 16/18 and other high-risk types. The likelihood of dramatically increased pathogenicity is low, since reduced screening frequencies should suffice to detect lesions caused by slowerprogressing non-16/18 types, and since our analysis conservatively assumes that all highrisk types progress as quickly as type 16. This is confirmed in the Webappendix, where a type-structured version of the model is developed for the purpose of understanding the potential impact of these compensatory factors. For example, under a scenario where type replacement causes a $10 \%$ increase in the prevalence of other high-risk types under $30 \%$ vaccine coverage, the percentage of infections by other high-risk types that lead to cervical cancer must increase by $240 \%$ for the screening threshold to exceed $x^{*}=0.61$.

Vaccination confers herd immunity, whereby unvaccinated members of the population experience reduced infection risk (29). Herd immunity is neglected in Equation (1), which further suggests that it provides an upper bound on the screening threshold. (However, some models indicate that herd immunity effects will be limited in the case of HPV vaccination (14)).

Equation (1) assumes that all women are screened and followed up in the same way. However, cervical screening programmes are often characterized by significant inequities. By comparison, school-based vaccination programmes tend to be more equitable. In the Webappendix we develop an equity-structured model for a population where all women have the same vaccine coverage but some women have a "low" screening rate and the remainder have a "high" screening rate. The equity-structured model predicts that, for a broad range of parameter values and the same average population screening rate, the threshold $x^{*}$ is lower in populations with more inequity. In summary, Equation (1) appears to provide a conservative estimate of how much screening adherence in vaccinated women can drop before total cervical cancer incidence in the population increases in the vaccine era. We note that the model makes the important assumption that vaccine-derived immunity does not wane. The long-term 
duration of immunity from the HPV vaccine is currently unknown, although it does show well-sustained protection for at least 7.5 years $(30,31)$. Likewise, the model does not explicitly include HPV transmission, age structure, disease natural history, or the time delay between HPV infection and onset of invasive cervical cancer. The strength of the model is its use of simplifying assumptions that tend to make its predictions conservative while also yielding a simple, transparent, and easily understood model.

\section{Conclusions}

Recent surveys of attitudes are consistent with the possibility of changing screening adherence in the vaccine era (see Webappendix for survey results) (32). Whether or not these changes will materialize remains to be seen. The analysis we have presented suggests that any decline in screening adherence in vaccinated women would have to be quite severe before cervical cancer incidence would increase in the vaccine era.

However, the required decline is somewhat less steep in populations that already have a highly effective cervical screening programme.

\section{Appendix: Derivation of model equations}

The differential equation that describes the rate of change in the population size of $n$ atrisk women over time $t$ is

(A1) $\frac{d n}{d t}=H-\left(r+s_{p r e}\right) n$

The equilibrium size $n_{\text {pre }}$ of the at-risk population in the pre-vaccine era is obtained by solving $d n / d t=0$ in Equation (A1), yielding

$$
n_{\text {pre }}=\frac{H}{r+s_{\text {pre }}} .
$$

According to this equation, the number of women at risk of developing cervical cancer increases with the infection rate $H$, and decreases if either the screening/follow-up rate $s_{\text {pre }}$ or the removal rate through other processes $r$ increase. In the vaccine era, under the assumptions of the main text, Equation (A1) becomes 
(A3) $\frac{d n}{d t}=H\left(1-f+f(1-e)-\left(r+s_{p o s t}(1-f+f x)\right) n\right.$,

and the corresponding equilibrium vaccine era population size is

(A4) $\quad n_{p o s t}=\frac{H(1-f e)}{r+s_{p o s t}(1-f+f x)}$.

The screening threshold $x^{*}$ below which $n_{\text {post }}>n_{\text {pre }}$ (policy resistance) can be obtained by solving $n_{\text {post }}=n_{\text {pre }}$, yielding Equation (1). Note that we constrain $x^{*}>0$.

Acknowledgements: The authors are grateful to Sanjay Basu for contributions to an earlier version of the manuscript, and to three anonymous reviewers for comments.

Role of funding source: This research was funded by the Canadian Institutes of Health Research, the Ontario Ministry of Research and Innovation, and the MacDonnell Foundation, who had no role in the research.

Author contributions: CTB designed the model, interpreted its output, and wrote the manuscript. ML and GC analyzed survey data. AG conceived of the study. All authors contributed to discussions and manuscript revisions.

Conflict of interest statement: CTB holds a research contract from, and has previously consulted for, GlaxoSmithKline. AG has previously consulted for Merck Research Laboratories. Neither company played a role in this research. 


\section{References}

1. Schmiedeskamp M, Kockler D. Human papillomavirus vaccines. Ann Pharmacother. 2006;40(7):1344-52.

2. Clifford G, Francheschi S, Diaz M, Munoz N, Villa LL. Chatper 3: HPV type distribution in women with and without cervical neoplastic diseases. Vaccine 2006;24 Suppl. 3:S26-S34.

3. Wright T, Goldie SJ. Screening for cervical cancer. Science 2000; 290(5497):1651.

4. Goldie SJ, Kim JJ, Myers ER. Cost-effectiveness of cervical cancer screening. Vaccine. 2006;24 Suppl 3:S164-70.

5. P Naucler, W Ryd, S Toernberg, A Strand, G Wadell, K Elfgren et al. Human papillomavirus and Papanicolaou tests to screen for cervical cancer. $N$ Eng J Med 2007; 357: 1589-1597.

6. M Mayrand, E Duarte-Franco, I Rodrigues, SD Walter, J Hanley, A Ferenczy et al. Human papilloamvirus DNA versus Papanicolaou screening tests for cervical cancer. N Engl J Med 2007; 357: 1579-1588.

7. Sterman J. Learning from evidence in a complex world. Am J Public Health 2006;96:505-14.

8. Goldie SJ, Grima D, Kohli M, Wright TC, Weinstein M, Franco E. A comprehensive natural history model of HPV infection and cervical cancer to estimate the clinical impact of a prophylactic HPV-16/18 vaccine. Int J Cancer 2003; 106(6):896-904.

9. Garnett GP, Kim JJ, French K, Goldie SJ. Chapter 21: Modelling the impact of HPV vaccines on cervical cancer and screening programmes. Vaccine 2006; 24 Suppl 3:S178-86.

10. Dasbach EJ, Elbasha EH, Insinga RP. Mathematical models for predicting the epidemiologic and economic impact of vaccination against human papillomavirus infection and disease. Epidemiol Rev 2006; 28:88-100.

11. Kim JJ, Kuntz KM, Stout NK, Mahmud S, Villa LL, Franco E, et al. Multiparameter Calibration of a Natural History Model of Cervical Cancer. Am J Epidemiol 2007; 166(2): 137-150. 
12. Elbasha EH, Dasbach EJ, Insinga RP. Model for Assessing Human Papillomavirus Vaccination Strategies. Emerg Infect Dis 2007;13(1).

13. Van de Velde N, Brisson M, Boily MC. Modeling human papillomavirus vaccine effectiveness: quantifying the impact of parameter uncertainty. Am J Epidemiol 2007;165(7):762-75.

14. Barnabas RV, Laukkanen P, Koskela P, Kontula O, Lehtinen M, Garnett G. Epidemiology of HPV 16 and cervical cancer in Finland and the potential impact of vaccination: mathematical modelling analyses. PLoS Medicine 2006;3(5):624-32.

15. Llamazares M, Smith? R. Evaluating human papillomavirus vaccination programs in Canada: should provincial healthcare pay for voluntary adult vaccination? $B M C$ Public Health 2008;8:114.

16. Basu S, Chapman G, Galvani A. Integrating epidemiology, psychology, and economics to achieve HPV vaccination targets. Proc Natl Acad Sci USA 2008;104:19018-23.

17. Taira AV, Neukermans CP, Sanders GD. Evaluating human papillomavirus vaccination programs. Emerg Infect Dis 2004;10(11):1915-23.

18. Goldie SJ, Kohli M, Grima D, Weinstein M, Wright TC, Bosch FX, et al. Projected Clinical Benefits and Cost-effectiveness of a Human Papillomavirus 16/18 Vaccine. J Natl Cancer Inst 2004;96(8):604-15.

19. Moscicki AB, Schiffman M, Kjaer S, Villa LL. Chapter 5: Updating the natural history of HPV and anogenital cancer. Vaccine 2006 Aug 21;24 Suppl 3:S42-51.

20. Goldhaber-Fiebert JD, Kim JJ, Kuntz KM, Salomon JA, Stout NK, Folse H, et al., editors. Calibrating a complex microsimulation model of human papillomavirus and cervical cancer to multiple data sources. Society for Medical Decision Making; 2006; Boston.

21. May RM. Uses and abuses of mathematics in biology. Science 2004; 303(5659):7903.

22. Dunne EF, Unger ER, Sternberg M, McQuillan G, Swan DC, Patel SS, et al. Prevalence of HPV Infection among Females in the United States. JAMA 2006; 297(8):813-9. 
23. Koutsky LA, Harper DM. Chapter 13: Current findings from prophylactic HPV vaccine trials. Vaccine 2006 Aug 21;24 Suppl 3:S114-21.

24. Sanjose S, Diaz M, Castellsague X, Clifford G, Bruni L, Munoz N et al. Worldwide prevalence and genotype distribution of cervical human papillmavirus DNA in women with normal cytology: a meta-analysis. Lancet Infect Dis 2007; 7: 453-59.

25. Insinga RP, Dasbach EJ, Elbasha EH, Liaw KL, Barr E. Progression and regression of incident cervical HPV 6, 11, 16 and 18 infections in young women. Infect Agent Cancer 2007; 2(1):15.

26. Insinga RP, Glass AG, Rush BB. Pap screening in a U.S. health plan. Cancer Epidemiol Biomarkers Prev 2004; 13(3):355-60.

27. GAVI Alliance: Vaccine Investment Strategy, 2009; Available from: http://www.gavialliance.org/vision/strategy/vaccine_investment/index.php.

28. Clifford GM, Smith JS, Plummer M. Human papillomavirus types in invasive cervical cancer worldwide: a meta-analysis. British J Cancer 2003;88:63-73.

29. Garnett GP. Role of Herd Immunity in Determining the Effect of Vaccines against Sexually Transmitted Disease. J Infect Dis 2005;191:S97-S106.

30. Harper DM, Franco EL, Wheeler CM, Moscicki AB, Romanowski B, Roteli-Martins CM. Sustained efficacy up to 4.5 years of a bivalent L1 virus-like particle vaccine against human papillomavirus types 16 and 18: follow-up from a randomised control trial. The Lancet 2006; 367(9518):1247-55.

31. Carvalho Nd, Roteli-Martins C, Teixeira J, Naud P, Borba Pd, Zahaf T. Immunogenicity and safety of HPV-16/18 AS04-Adjuvanted vaccine up to 7.3 Y. Published Abastract, 25th International Papillomavirus Conference, May 8-14 2009, Malmo, Sweden. 2009.

32. Sauvageau C, Duval B, Gilca V, Lavoie F, Ouakki M. Human papillomavirus vaccine and cervical cancer screening acceptability among adults in Quebec, Canada. BMC Public Health 2007; 7:304. 
Figure 1: Screening threshold $x^{*}$ from Equation (2) under the "opportunistic screening" scenario, as a function of (a) vaccine effectiveness $e$, (b) rate of removal through screening and follow-up $s$, (c) rate of removal through other processes $r$. Arrows denote baseline parameter values $e=0.14, s=0.44 /$ year, $r=0.83 /$ year.
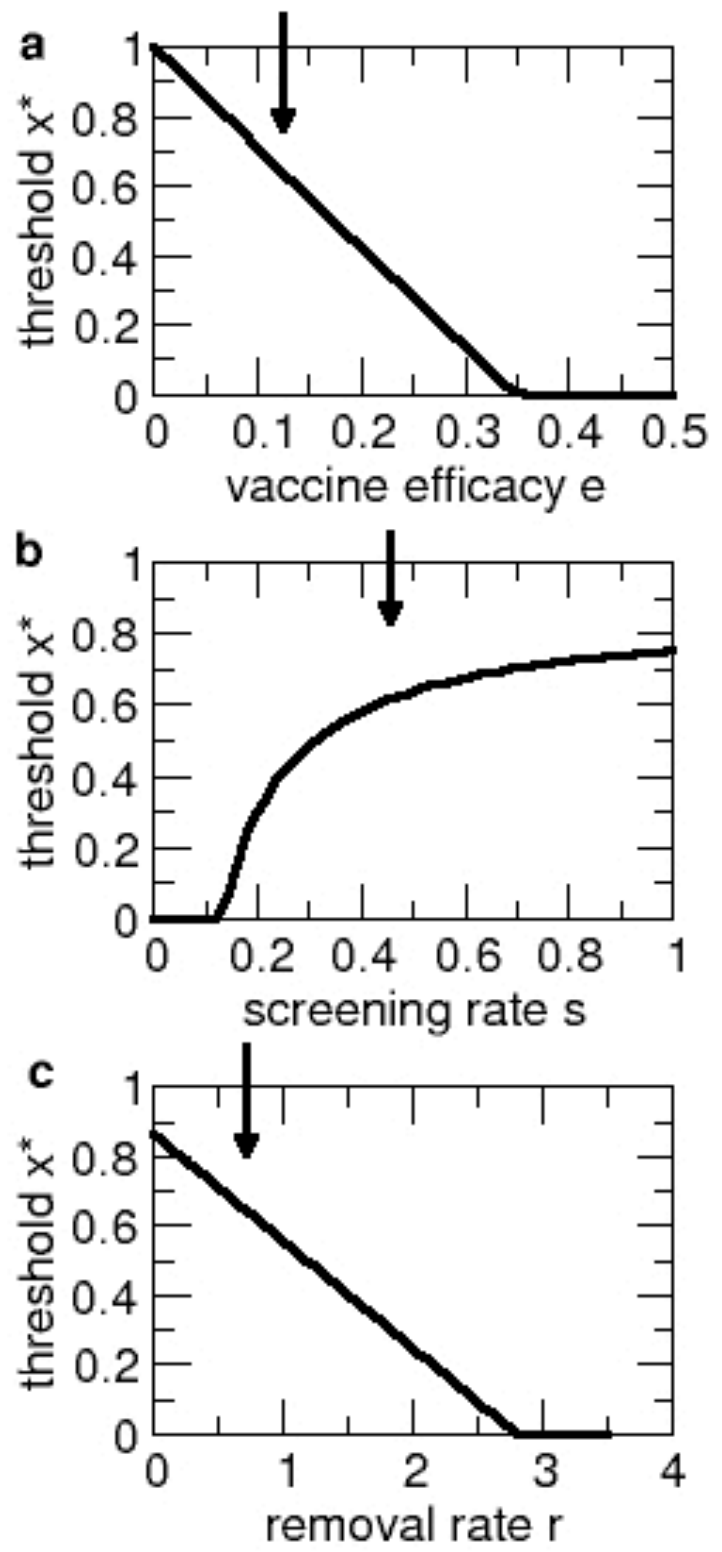
Table 1: Results of probabilistic uncertainty analysis for three screening scenarios.

\begin{tabular}{|c|c|c|c|}
\hline & "Limited screening" & "Opportunistic screening" & "Organized screening" \\
\hline & \multicolumn{3}{|c|}{ Parameter ranges for uncertainty analysis } \\
\hline \multirow{2}{*}{$\begin{array}{l}\text { Pre-vaccine screening } \\
\text { removal rate } s_{\text {pre }}\end{array}$} & $(0.15,0.25)$ & $(0.4,0.5)$ & $(0.70,0.80)$ \\
\hline & \multicolumn{3}{|c|}{ Simulated screening threshold $x^{*}$ for low vaccine coverage, $f=0.3$} \\
\hline Median & 0 & 0.20 & 0.33 \\
\hline \multirow[t]{2}{*}{ Range* } & $(0,0.22)$ & $(0,0.57)$ & $(0,0.69)$ \\
\hline & \multicolumn{3}{|c|}{ Simulated screening threshold $x^{*}$ for high vaccine coverage, $f=0.7$} \\
\hline Median & 0 & 0.38 & 0.51 \\
\hline Range* & $(0,0.30)$ & $(0.04,0.72)$ & $(0.26,0.77)$ \\
\hline
\end{tabular}

* Median +/- 2 standard deviations. 